Revista de Estudios Histórico-Jurídicos

[Sección Noticias]

XXXII (Valparaíso, Chile, 2010)

[pp. $651-656$ ]

\title{
"IN MEMORIAM" Pablo Fuenteseca (1922-2009)
}

"Una de las grandes satisfacciones que pueden caber a un discípulo es la de honrar públicamente a su maestro en el camino científico", escribía Pablo Fuenteseca en el año 1968, con ocasión de su participación en el homenaje tributado al profesor Álvaro d'Ors, para conmemorar el vigésimo quinto aniversario de su acceso a la cátedra. Con análogo espíritu se redactan las páginas que siguen, desde el profundo afecto y reconocimiento que siempre profesé a D. Pablo Fuenteseca, como maestro y figura señera que fue de la ciencia romanística española e internacional.

Pablo Fuenteseca nació en Cospeito, provincia de Lugo, el 31 de julio de 1922 y murió en Madrid, el 29 de octubre de 2009, a los 87 años de edad. Su destacada personalidad se manifestó ya desde sus primeros estudios de bachillerato realizados en el Instituto de Lugo. A uno de sus profesores en aquella sede, el renombrado historiador e intelectual galleguista Filgueira Valverde, le oí personalmente decir que Pablo Fuenteseca era uno de sus orgullos docentes. Asimismo han sido muchas las ocasiones en las que oí rememorar a mi maestro, con satisfacción, sus años en el Instituto, cuyos pasillos volvimos a recorrer con añoranza varias veces, en alguna estancia compartida en la capital lucense. También le agradaba resaltar el alto nivel de calidad de la enseñanza impartida en sus aulas, lo que puedo asimismo corroborar como alumno que fui, treinta años después, del mencionado centro docente.

En la Universidad de Santiago de Compostela, se licenció Pablo Fuenteseca en Derecho, con premio extraordinario, y en Filosofía y Letras, sección de Historia y se graduó en Estudios Sociales, lo que le llevó a impartir docencia en la Escuela Social de Santiago y a desempeñar la ayudantía de la cátedra de Historia Antigua de la Facultad de Filosofía y Letras. En 1945, en Santiago, concluida la licenciatura en Derecho, se acogió al magisterio de D. Alvaro d'Ors, ya entonces uno de los más eminentes romanistas españoles, que se había incorporado, en ese curso académico, a la Universidad compostelana, por concurso de traslado desde la Universidad de Granada.

En 1947 el profesor Fuenteseca obtuvo, por oposición, la plaza de profesor adjunto de Derecho romano en la Facultad de Derecho de Santiago y, a partir de 
esta fecha, completa su formación jurídica en prestigiosos centros de investigación y universidades europeas. Fue becario del Instituto Nacional de Estudios Jurídicos de Madrid y, desde 1949, colaborador y secretario general de su sección en Santiago. En el curso 1950-1951, becado por la Dirección General de Relaciones Culturales, del Ministerio de Asuntos Exteriores, amplió estudios en el Istituto di Diritto Romano de la Universitá La Sapienza de Roma. Durante los años 1953 a 1956 como becario de la Fundación Alexander von Humboldt, en la Universidad de Heidelberg trabajó con el egregio romanista profesor Wolgang Kunkel, a quien siempre consideró su segundo maestro, y con quien volvió a colaborar en 1958, becado por la Dirección General de Enseñanza Universitaria, en el Instituto Leopold Wenger de la Universidad de Munich. En el año 1961, becado por el Institut de Droit Romain, realizó una estancia en la Universidad de la Sorbona, en París. A las sesiones de Heidelberg, en los años de la década iniciada en 1950 se refiere Fuenteseca, en la "Introducción" de sus Investigaciones de Derecho procesal romano, con unas líneas de homenaje del siguiente tenor: "A aquellas horas germánicas, al maestro Kunkel que las presidió con sabiduría y auctoritas y a quienes fueron compañeros de la tarea revisionista del proceso romano (Broggini, Jahr, Kelly y, más fugazmente Nörr, Sturm, Kiefner y Wubbe) va mi recuerdo nostálgico con estas páginas de Derecho procesal romano".

En el año 1956 alcanzó Fuenteseca, por oposición, con el número uno de las dos plazas convocadas, la cátedra de Derecho Romano de la Universidad de La Laguna, en la que permaneció hasta 1960. De aquella época recordaba, de manera especial, la amistad entablada con el profesor González Vicén, a la sazón catedrático de Filosofía del Derecho, con quien tuvo ocasión de compartir sus comunes vivencias y conocimientos de la cultura germana, y las largas sesiones de estudio en la Facultad. Entre los años 1960 y 1970 desempeñó la cátedra de Derecho romano de la Universidad de Salamanca, de cuya Facultad de Derecho llegó a ser Decano. Fue también profesor de Instituciones romanas de la Facultad de Filosofía y Letras de la Universidad Salmantina. En 1970, en virtud de un concurso de méritos, fue nombrado catedrático y director del Departamento de Derecho Romano de la Universidad Autónoma de Madrid, destino en el que permaneció hasta su jubilación en 1987, a los 65 años de edad. El inesperado recorte en cinco años de la edad de jubilación prevista, establecido por una disposición legal, supuso uno de los mayores contratiempos en la trayectoria académica del profesor Fuenteseca, que había consagrado su vida a la Universidad y veía frustrada su legítima expectativa de jubilación a los 70 años, encontrándose en plenitud de facultades y con múltiples proyectos pendientes.

Fue D. Pablo Fuenteseca uno de los fundadores de la Facultad de Derecho de la Universidad Autónoma, junto con, entre otros ilustres profesores, D. Aurelio Menéndez, que fue su primer y emblemático Decano, y la persona clave en la conformación de su claustro académico, D. Gonzalo Rodríguez Mourullo, que desempeño el segundo decanato; y D. Luis Díez-Picazo, y contribuyó de forma decisiva a su consideración, desde el momento fundacional, como la más prestigiosa Facultad de Derecho española. En esta etapa de su vida académica, fue nombrado, por resolución rectoral, director del Colegio Universitario de Cuenca, adscrito a la 
Universidad Autónoma de Madrid, institución a la que llamó a colaborar, como subdirectores, en periodos sucesivos, a sus discípulos, Jesús Daza y a quien firma estas líneas.

Fue D. Pablo Fuenteseca un maestro de los de verdad, de los pocos que logran consolidar una amplia y prestigiosa escuela. Generoso con sus discípulos, nos enseñó a razonar, nos dedicó mucho tiempo, reforzó nuestra vocación intelectual con su inestimable ejemplo de trabajador incansable, compartió con nosotros su inmensa cultura, nos enseñó a distinguir entre las conjeturas y los errores, y dirigió la carrera académica y científica de quienes nos acogimos a su magisterio, con integridad, sin escatimar esfuerzos y con notable acierto. Fruto de todo ello fue la constitución de la escuela más numerosa de la romanística española. En sus años salmantinos accedieron a la cátedra sus primeros discípulos, Alfredo Calonge y Armando Torrent. A su etapa en la Universidad Autónoma de Madrid, corresponde el despertar de las vocaciones universitarias, la formación y la dirección del cursus honorum, hasta el acceso a la cátedra, de Antonio Ortega, Justo García Sánchez, Jesús Daza, Antonio Fernández de Buján, Esteban Varela, Amparo González y Gustavo de las Heras. Numerosos profesores titulares y doctores, se beneficiaron asimismo, de forma directa, del magisterio de Pablo Fuenteseca.

Si bien quizás no sea éste el momento más adecuado para hacer una valoración específica de sus aportaciones concretas como romanista, sí cabe subrayar que, como autor de una obra extensa y rigurosa, sus estudios monográficos abarcan los más diversos campos de estudio del Derecho romano y que la agudeza exegética de sus opiniones sobre los textos, sus certeros análisis de los temas tratados y los resultados de sus investigaciones, plenas de aportaciones originales, constituyen un punto de referencia necesario para estudios posteriores.

De entre sus obras de conjunto, cabe resaltar: su Historia del Derecho romano y el Derecho privado romano. El primero de estos manuales, publicado en 1987, y que puede considerarse una de las cumbres en su producción científica, constituye la culminación de su primera visión global de la experiencia constitucional romana, que publicó en Salamanca en 1963, bajo el título de Lecciones de Historia del Derecho romano y fue objeto de numerosas ediciones y reimpresiones. Su Derecho privado romano, publicado en 1978, es una obra que puede calificarse de maestra en su género. Escrita con ejemplar rigor y un estilo sobrio y pulido, con sello propio y pleno de matices, resulta original, armoniosa y sistemática en la exposición de las instituciones, a las que infunde vida propia con su personal visión de la experiencia iusprivatística romana.

De entre los temas de investigación, objeto de su predilección, cabría referirse a los siguientes:

a) Derecho público romano, al que entregó permanente dedicación, fruto de la cual son, entre otras aportaciones, además de sus manuales de historia constitucional romana, estos escritos:

i) Oriente y Occidente en la Compilación de Justiniano, en Información Jurídica, 109 (Ministerio de Justicia, 1952), pp. 547-559.

ii) Historia y dogmática en la jurisprudencia europea, en Revista de Derecho Notarial, 10 (Madrid, 1956), pp. 1-22. 
iii) La “auctoritas" como principio constitucional romano, en Estudios en Homenaje al Prof. Albaladejo (2004), I, pp. 1763-1774.

b) El sustrato romanístico del Derecho europeo, frente a la idea de los nacionalismos jurídicos; así en:

i) La "Lección inaugural" del curso 1977-1978 de la Universidad Autónoma de Madrid, titulada: El gran dilema histórico del pensamiento jurídico europeo: ¿ley natural o ley politica? (Madrid, 1978).

ii) La recepción de la idea imperial en la edad media española y sus raices romanas, en Studi Sassaress, 8 (1980-1981), pp. 63-97 = Estudios en Homenaje al Prof. Juan Iglesias (Madrid, 1988), II, pp. 747-771.

iii) De la res publica romana al Estado Moderno, en Journées Internationales d'Histoire du Droit (Bilbao, Universidad del País Vasco, 1992), pp. 55-92.

iv) El Derecho Romano y la Historia del Derecho Europeo, en Actas del IV Congreso Iberoamericano de Derecho Romano (Universidad de Vigo, 1998), II, pp. 303-312.

v) La formación greco-romana del actual concepto europeo del Estado. Discurso de Ingreso en la Real Academia de Jurisprudencia y Legislación leido el 21 de junio de 2004 y contestado por el profesor Fairen Guillén (Madrid,2004).

c) Sus investigaciones sobre Derecho procesal romano, así en:

i) ¿Existió la denominada "legis actio sacramento in personam"? en AHDE., 25 (1955), pp. 543- 566.

ii) Las "legis actiones" como etapas del proceso romano, en AHDE., 34 (1964), pp. 209-233.

iii) La necesidad de retornar al estudio del edicto pretorio, en AHDE., 39 (1969), pp. 69-92.

iv) Reflexiones sobre la tricotomia “actio petitio persecutio", en AHDE., 40 (1970), pp. 123-226.

d) El Derecho privado romano cuyas investigaciones sobre el cual abarcan multitud de temas específicos, entre los que cabe destacar, sus estudios acerca de:

i) La idea de contrato, como en: Los sistemas expositivos de las obligaciones contractuales en la jurisprudencia romana y la idea de "contractus", en AHDE., 23 (1953), pp. 539-577; Origen del concepto romano de obligación ("obligatio"), en Libro Homenaje a Roca Sastre (Madrid, Junta de Decanos de Colegios Notariales, 1976), I, pp. 11-172; y La "obligatio" como "vinculum iuris (civilis)" y la "obligatio rei pignoris causa", en "Iuris Vincula". Studi in onore di Mario Talamanca (2001), pp. 441-503.

ii) La noción de posesión, así en: "Possessio domino ignorante", en $A H D E ., 24$ (1954), pp. 559-585.

iii) Las ideas de "mancipium”, “dominium”, "proprietas”, así en: "Mancipium”, "mancipatio", “dominium”, en Labeo, 4 (1958), pp. 135-149; Trasferimento della propietá e "auctoritas" nella vendita romana, en Atti del Congresso Internazionale: "Vendita e trasferimento della proprietá nella prospettiva storico-comparatistica" (Milano, Giuffré, 1991), pp. 73-118; y El "titulus" y el "modus" de los glosadores boloñeses. Clave interpretativa del asindeton "usus auctoritas" (XII Tab. VI,3) y de la historia del concepto romano de la propiedad, en Estudios jurídicos en homenaje al 
profesor Luis Diez-Picazo (2003), IV, pp. 5727-5755.

iv) La noción de herencia, así en Puntos de vista de la Jurisprudencia romana respecto a la "hereditas iacens", en AHDE., 26 (1956), pp. 243-267.

e) La metodología y las aportaciones marxistas en el marco de la investigación romanística, como en:

i) La comunicación al "Symposium sobre Marxismo y Derecho romano", celebrado en la Fundación Pastor de Estudios Clásicos, en marzo de 1977.

ii) La tesis doctoral dirigida al profesor Viñas Otero, bajo el título: Función del Tribunado de la Plebe: ¿Reforma política o revolución social? (Madrid, 1983).

f) Temática gallega:

i) O Dereito Civil de Galicia: Consideraciones histórico-xurídicas (Orense, Universidad de Vigo, 1996), pp. 14-25, que constituyó la lección magistral pronunciada con ocasión de su nombramiento como miembro honorario del Claustro de Profesores de la Facultad de Derecho de la Universidad de Vigo, en enero de 1996.

ii) La comunicación presentada al "Primer Congreso Gallego de Estudios Clásicos”, celebrado en Santiago en 1979, con el título La sede compostelana y la recepción de la idea imperial en España.

iii) La tesis doctoral dirigida al profesor Salgado Novoa, bajo el título: El Foro y la evolución de la propiedad en Galicia.

iv) La tesis doctoral dirigida a D. J. C. Paz Ares, bajo el título: Instituciones al servicio de la casa en el Derecho Civil de Galicia (Salamanca, 1964).

Todas sus investigaciones, las anteriormente citadas y los restantes, recopilados en la Colección de estudios, que edita el Centro de Estudios del Colegio de Registradores de la Propiedad y Mercantiles de España, y coordinada por sus hijas, Cristina y Margarita Fuenteseca Degeneffe, son un modelo de análisis históricojurídico de los textos y de reconstrucción de los dogmas de Derecho privado y Derecho público romano. Fue partidario del estudio de la proyección histórica de la dogmática romana hasta el Derecho actual, y conforme a este método de investigación ("Dogmengeschichte"), escribió varias de sus aportaciones y se realizaron algunas de las dieciocho tesis doctorales defendidas bajo su dirección.

La historia de la relación personal y académica con mi maestro se remonta a 1972, en el que fui nombrado, a los 18 años, y a propuesta suya, alumno interno del Departamento de Derecho Romano de la Universidad Autónoma de Madrid. Son muchas, desde entonces, las vivencias compartidas, a lo largo de dilatados años de estrecha relación discipular y personal, muchas las jornadas convividas de más de diez horas diarias en la Facultad, a lo largo de más de una década, y muchas las enseñanzas recibidas sobre la Romanística y la Universidad que le tocó vivir. Mis estancias de varios días en su Cospeito natal para visitar a su madre,

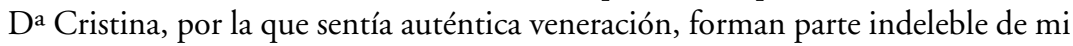
trayectoria vital.

Mi primera monografía, publicada en 1981, y realizada bajo su dirección, sobre el Filiusfamilias de vida independiente en Roma y en el Derecho fiscal español, se la dediqué a mi maestro, con las siguientes palabras: "Al dedicar mi primer estudio extenso al Profesor Pablo Fuenteseca, cumplo con profunda satisfacción la obligatio naturalis que a lo largo de más de una década tengo contraída con mi 
maestro. Su palabra, su ejemplo y su ayuda han sido, en todo momento, factores decisivos, en mi vocación y en mi formación como romanista”. Cuando, quince años después, en 1996, publiqué la primera edición de mi Derecho público romano, procedí a incluir un último párrafo del prólogo -que se mantiene en las posteriores ediciones aparecidas desde entonces- de reconocimiento de deuda con mi maestro, expresada conforme al siguiente tenor: "Quiero finalmente dejar constancia de la labor de impulso, en el ámbito tanto de la docencia como de la investigación, realizada en materia de Derecho público romano, por mi maestro, el Profesor Pablo Fuenteseca, algunas de cuyas enseñanzas, laten o se recogen en las explicaciones contenidas en este manual".

Tenía D. Pablo Fuenteseca fe en el Derecho y en la obra educadora y civilizadora del mundo clásico. Le gustaba ejercer su magisterio en la conversación cotidiana. Jurista prestigioso y figura señera del romanismo, fue miembro de muchas y autorizadas sociedades científicas españolas e internacionales. Dotado de una gran inteligencia y agudeza, sobrio y riguroso, procuraba razonar siempre su argumentación y fundamentar sus decisiones, ajenas a cualquier clase de formalismo conceptual. Trabajador infatigable, practicó la cultura del esfuerzo a lo largo de toda su dilatada y fecunda vida, habiéndole oído en muchas ocasiones ponderar el inestimable valor que tiene lo conseguido mediante el esfuerzo personal, conforme a la máxima machadiana, a la que yo aludía siempre, por indicación suya, en mis oposiciones. Su vocación intelectual acentuó su sentido crítico y dio sentido a su permanente vocación de saber. Fueron sus señas de identidad, al margen de su familia, que constituyó siempre su primera preocupación, el Derecho romano, la Universidad, Galicia en todas sus manifestaciones, y la formación de una escuela de romanistas a la que dedicó muchos afanes y muchas horas de su vida.

Fue D. Pablo Fuenteseca una persona de una cultura inmensa, alimentada por constantes lecturas de obras históricas, filosóficas y políticas. Universitario cabal, su integridad moral y su nobleza quedarán como referente para las generaciones futuras. Modélico padre de familia, su esposa Da Margarita Degeneffe, con su inteligencia y su bondad, ha sido la persona clave en su equilibrio vital. Sus hijas Cristina, profesora titular de Derecho civil y Margarita, catedrática de Derecho romano, le han dado muchas satisfacciones. La obtención de la cátedra por su hija Margarita, en su misma disciplina, le produjo una especial alegría.

La comunidad universitaria, nacional e internacional ha sido testigo del altísimo nivel como romanista y como jurista alcanzado por el maestro, y de su insobornable honradez académica.

Desde el afecto profundo de discípulo que tuvo la fortuna de mantener durante muchos años una relación casi filial con su maestro, y con el reconocimiento, admiración y agradecimiento, que sentimos hacia su persona y su obra todos los que nos hemos formado a su lado, sirvan estas páginas para rendir un sentido y modesto homenaje a la imperecedera memoria de D. Pablo Fuenteseca. 